\title{
Wearable Sensor-Based In-Home Assessment of Gait, Balance, and Physical Activity for Discrimination of Frailty Status: Baseline Results of the Arizona Frailty Cohort Study
}

\author{
Michael Schwenk ${ }^{\mathrm{a}, \mathrm{c}}$ Jane Mohler ${ }^{\mathrm{a}-\mathrm{d}}$ Christopher Wendel ${ }^{\mathrm{a}, \mathrm{c}}$ \\ Karen D'Huyvetter ${ }^{a, b}$ Mindy Fain ${ }^{a-c}$ Ruth Taylor-Piliae ${ }^{a, b}$, d Bijan Najafi ${ }^{a-c}$ \\ ${ }^{a}$ Arizona Center on Aging, ${ }^{b}$ Division of Geriatrics, General Internal Medicine and Palliative Medicine, \\ Department of Medicine, ' Interdisciplinary Consortium on Advanced Motion Performance (iCAMP), Department of \\ Surgery, College of Medicine, and ${ }^{\mathrm{d}}$ College of Nursing, University of Arizona, Tucson, Ariz., USA
}

\section{Key Words}

Frailty · Wearable sensors · Monitoring · Physical function .

Physical activity

\begin{abstract}
Background: Frailty is a geriatric syndrome resulting from age-related cumulative decline across multiple physiologic systems, impaired homeostatic reserve, and reduced capacity to resist stress. Based on recent estimates, $10 \%$ of community-dwelling older individuals are frail and another $41.6 \%$ are prefrail. Frail elders account for the highest health care costs in industrialized nations. Impaired physical function is a major indicator of frailty, and functional performance tests are useful for the identification of frailty. Objective instrumented assessments of physical functioning that are feasible for home frailty screening have not been adequately developed. Objective: To examine the ability of wearable sensor-based in-home assessment of gait, balance, and physical activity (PA) to discriminate between frailty levels (nonfrail, prefrail, and frail). Methods: In an observational cross-sectional study, in-home visits were completed in 125 older adults (nonfrail: $n=44$, prefrail: $n=60$, frail: $n=21$ ) living in Tucson, Ariz., USA, between September 2012 and November 2013. Temporal-spatial gait parameters (speed,
\end{abstract}

stride length, stride time, double support, and variability of stride velocity), postural balance (sway of hip, ankle, and center of mass), and PA (percentage of walking, standing, sitting, and lying; mean duration and variability of single walking, standing, sitting, and lying bouts) were measured in the participant's home using validated wearable sensor technology. Logistic regression was used to assess the most sensitive gait, balance, and PA variables for identifying prefrail participants (vs. nonfrail). Multinomial logistic regression was used to identify variables sensitive to discriminate between three frailty levels. Results: Gait speed (area under the curve, AUC $=0.802$ ), hip sway (AUC $=0.734)$, and steps/day (AUC $=0.736$ ) were the most sensitive parameters for the identification of prefrailty. Multinomial regression revealed that stride length (AUC $=0.857$ ) and double support (AUC = 0.841 ) were the most sensitive gait parameters for discriminating between three frailty levels. Interestingly, walking bout duration variability was the most sensitive PA parameter for discriminating between three frailty levels (AUC =

Some parts of this study have been presented at the 2013 Annual Scientific Meeting of the American Geriatric Society, the 2012 Annual Scientific Meeting of the Gerontological Society of America, and the ICFSR 2014 International Conference on Frailty and Sarcopenia Research.

\section{KARGER 125}

C 2014 S. Karger AG, Base

0304-324X/14/0613-0258\$39.50/0

E-Mail karger@karger.com

www.karger.com/ger
Jane Mohler, NP-C, MPH, PhD

Arizona Center on Aging, College of Medicine University of Arizona

1821 E. Elm Street, Tucson, AZ 85719 (USA)

E-Mail jmohler@aging.arizona.edu 
0.818). No balance parameter discriminated between three frailty levels. Conclusion: Our results indicate that unique parameters derived from objective assessment of gait, balance, and PA are sensitive for the identification of prefrailty and the classification of a subject's frailty level. The present findings highlight the potential of wearable sensor technology for in-home assessment of frailty status.

(c) 2014 S. Karger AG, Basel

\section{Introduction}

Frailty is a geriatric syndrome resulting from age-related cumulative decline across multiple physiologic systems, impaired homeostatic reserve, and reduced capacity to resist stress [1]. Frailty increases vulnerability towards adverse health outcomes including falls, hospitalization, institutionalization, and mortality [1]. Based on recent estimates, $10 \%$ of community-dwelling older individuals are frail and another $41.6 \%$ are prefrail [2]. Frail elders account for the highest health care costs in industrialized nations [2].

One of the most commonly accepted operational definitions of frailty is the classification proposed by Fried et al. [1] (i.e., weight loss, weakness, exhaustion, slowness, and low energy expenditure). However, its use may have limited feasibility and reliability in a routine care setting [3-6]. The criteria of weight loss, exhaustion, and energy expenditure are usually self-reported measures and may be prone to bias $[3,5,6]$. An objective frailty screening tool may be more appropriate for routine assessment.

Impaired physical function is a major indicator of frailty [1], and measures of functional performance are useful frailty screening tools [3,7-12]. Most studies have used subjective or semiobjective (i.e., stopwatch) assessments [3, 10-12], despite limitations including self-report bias and nonobjective parameters. For instance, the Vulnerable Elders Survey [10] includes self-rating of functional status (among other items), but a potential overestimation of physical competence has been discussed as limitation [10, 13]. Frailty screening using the Short Physical Performance Battery $[3,12]$ has acceptable reliability for the 4 -meter walk and 5 sits-to-stand test, but may have limited reliability for the balance subscale, based on the use of a stopwatch [14]. Using an objective stabilometry measure [15] instead of a stopwatch may allow quantification of more sensitive balance parameters for identifying frailty; however, to our knowledge, this has not been adequately explored.

Slow gait speed has been reported as the most easily identifiable feature of frailty $[1,16]$, but it does not pro-

Wearable Sensor-Based Assessment of Frailty vide other temporal-spatial gait characteristics which may have a strong association with frailty $[9,11]$. A recent systematic review reported that frailty is associated with low performance in several temporal-spatial gait parameters beyond speed, including high stride time variability, reduced step length, and increased double support [11]. However, it remains unclear if gait parameters can improve frailty screening in comparison to using gait speed only. Additionally, studies using quantitative gait assessment in frail individuals have been largely conducted in laboratory or clinical environments using camera systems $[17,18]$, force platforms [18], or electronic carpets $[9,19]$, and are not fully translatable to the home and community. It remains unclear if the objective assessment of gait characteristics is feasible at home and if it can increase the accuracy of frailty screening.

In addition to impairment in physical function, a low level of self-reported physical activity (PA) is a key indicator of frailty [16], and an increased level of PA may prevent or even reverse frailty [20]. Whereas frailty-associated functional performance parameters (e.g., reduced gait speed and strength deficits) have been identified $[3,7,8]$, frailty-specific 'natural' PA characteristics remain to be elucidated [21]. Self-report questionnaires may not be suitable to document frailty-related PA characteristics due to limited validity in measuring low intensity activities of daily living (ADL) [22] - the most prevalent activities in frail older adults [23]. In contrast to self-report, objective PA assessment using wearable sensors can provide precise documentation of everyday activities including walking, standing, sitting, and lying [24, 25], and in turn, may allow the identification of frailty-specific PA patterns in the home and community. Different frailty levels may be characterized by differences in everyday PA patterns, such as reduced distances walked continuously (e.g., due to exhaustion and diminishing strength) or reduced complexity of PA. Loss of complexity in the dynamics of physiologic systems (i.e., heart rate, hormonal rhythms, and postural sway) have been associated with frailty [26] and may also be reflected by a less variable PA pattern. However, to our knowledge, previous studies have used step number only for objectively assessing frailty [21], but did not quantify more specific everyday PA characteristics. In recent years, advances in wearable sensor technology have provided a new avenue for measuring both physical function $[15,27,28]$ and PA [24, 25] across varying populations. Wearable sensors have the benefits of objectivity, portability, and low cost [28], making these devices useful for frailty assessment in the home and community. 
The purpose of the Arizona Frailty Cohort Study is to identify relevant sensor-based markers of physical function (i.e., deficits in gait and balance) and everyday PA (i.e., changes in walking, standing, sitting, lying, and transfer characteristics) useful for home-based frailty screening. This study reports the baseline results and explores the capability of parameters to discriminate crosssectionally between nonfrail, prefrail, and frail categories. The confirmatory aim of this study was to evaluate the ability of sensor-based home assessment to identify prefrailty and frailty based on established outcomes (i.e., gait speed). We hypothesized that we could separate frailty groups (nonfrail, prefrail, and frail) through sensor-based assessment of gait speed. The exploratory aim was to identify new objective parameters among gait, balance, and PA measures, which might increase the accuracy of frailty assessment.

\section{Methods}

We performed an observational cross-sectional descriptive study within a large Southwestern academic medical center, affiliated with a statewide Center on Aging located in Tucson, Ariz., USA. Tucson has an estimated 152,000 older adults, with an estimated 16,700 residents (11\%) who have clinical frailty syndrome. Participants were recruited from primary, secondary, and tertiary health care settings within our large and highly affiliated academic network as well as from community providers and aging service organizations. In-home baseline visits were completed between September 2012 and November 2013.

\section{Participants}

Adults aged $\geq 65$ years and without gait or mobility disorders who reported being able to walk $\geq 9.14 \mathrm{~m}$ (30 feet) with or without an assistive device were eligible to be screened for study entry. Exclusion criteria included a Mini-Mental State Examination (MMSE) [29] score $<23$, terminal illness, or unwillingness to participate. Eligible subjects signed a written informed consent form approved by the Institutional Review Board of the University of Arizona. The clinical trial was registered with ClinicalTrials.gov, identifier NCT01880229.

\section{Measures}

Demographic and Clinical Characteristics

A team of two trained clinical coordinators visited patients within their home or assisted living setting for collecting data. Measures included self-reported history of falls, use of assistive device (yes/no), and number of prescriptions. Height was obtained by a tape measure, weight was measured using a bathroom scale (Ozeri Touch II; Ozeri ${ }^{\mathrm{TM}}$, San Diego, Calif., USA), and BMI was calculated based on height and weight. Interviewer-administered questionnaires included the MMSE, Mobility-Tiredness Scale [30], Center for Epidemiologic Studies Depression Scale (CES-D) [31], Falls Efficacy Scale-International [32], and Barthel ADL Scale [33].

\section{Assessment of Frailty Level}

Frailty was operationalized using the five components proposed by Fried et al. [1]. Weight loss was evaluated by self-reported unintentional weight loss of $>4.54 \mathrm{~kg}$ (10 pounds) over the past year. Weakness was measured by the grip strength test using a hydraulic hand dynamometer (Fabrication Enterprises Inc., Elmsford, N.Y., USA). Three measures were taken, and the arithmetic mean was used to identify this criterion. Weakness was defined according to sex and the BMI cutoffs used by Fried et al. Exhaustion was quantified by two statements of the CES-D questionnaire [31]: 'I felt everything I did was an effort' and 'I could not get going'. The frequency of 'occasionally' or 'most of the time' as a reply to either of these statements was considered as an indication of exhaustion [1]. Slowness was quantified by a walking time of $4.57 \mathrm{~m}$ (15 feet at the usual pace) measured by a stopwatch and stratified by gender and height using the cutoffs defined by Fried et al. Low energy expenditure was measured based on the short version of the Minnesota Leisure Time Activity questionnaire [34], as proposed by Fried et al. Calories expended per week were calculated according to the questionnaire's manual, and gender-stratified cutoff for low activity was used as described by Fried et al.

Participants were scored one point for each criterion found, totaling a score that could range from 0 to 5 . Frailty level was categorized following Fried et al. [1]: nonfrail = no criteria; prefrail = one or two criteria, and frail = three or more criteria. Norm-based scoring was performed using a computerized scoring algorithm (Frailty Assessment Tool; The Johns Hopkins Center on Aging and Health, Baltimore, Md., USA), which is based on the criteria of Fried et al.

Sensor-Based Assessment of Gait and Balance

We used commercially available technology for gait assessment (LEGSys $^{\mathrm{TM}}$ : Locomotion Evaluation and Gait System; BioSensics, Cambridge, Mass., USA) and balance assessment (BalanSens ${ }^{\mathrm{TM}}$; BioSensics). Both systems use the same hardware configuration of five small inertial sensors attached to the shanks, thighs, and lower back. Each sensor module includes a triaxial accelerometer, magnetometer, and gyroscope (sample frequency $100 \mathrm{~Hz}$ ). Different software was used for extraction of gait parameters (LEGSys) and balance parameters (BalanSens), which are based on validated algorithms [15, 27, 28].

Gait Assessment. Gait assessment was conducted under singleand dual-task (counting backward by 1 , starting from 100) conditions. Participants walked a distance of $4.57 \mathrm{~m}$ (15 feet) at a selfselected speed under each condition in their home. Where possible, we assessed gait without a walking aid. Temporal-spatial gait parameters [i.e., speed, stride time, stride length, double support (as percentage of stride time), and gait variability defined as coefficient of variation $(\mathrm{CV})$ of stride velocity] were extracted from the raw data using validated algorithms that have been described previously in detail $[27,28]$. To summarize, the gait phases were determined from the precise moments of heel-strike (initial foot contact) and toe-off (terminal foot contact). These moments were extracted from gyroscopes attached to each shank through a local minimal peak detection scheme [27]. Based on the subject's height and using a two-link inverse pendulum model, temporal-spatial gait parameters were estimated by integrating the angular rate of rotation of the thigh and shank [27].

Balance Assessment. Balance was measured during $15 \mathrm{~s}$ standing with feet close together and eyes closed. Postural sway param- 
eters including sway of ankle, hip, and center of mass (CoM) in medial-lateral (ML) and anterior-posterior (AP) direction were extracted from the raw data using validated algorithms, as described elsewhere in detail [15]. To summarize, data from two sensors (lower back and shank) were used to estimate three-dimensional angles of the hip and ankle joints. Each sensor provided real-time quaternions that were subsequently converted to Euler angles. The resulting three-dimensional angles were used to estimate the trajectory of the subject's ankle and hip. A two-segment model of the body was used to calculate CoM range of motion in AP and ML direction [15]. The area of sway for CoM was calculated by multiplying the range of motion in ML and AP directions after excluding outliers, which were estimated by calculating the 5 th and 95th percentiles of data [15].

Sensor-Based PA Assessment

PA was quantified during a 24-hour period by a motion sensor (PAMSys ${ }^{\mathrm{TM}}$; BioSensics) inserted into a T-shirt, with a device pocket located at the sternum. The PAMSys is a small $(5.1 \times 3 \times$ $1.6 \mathrm{~cm}$ ), light (24 g), long-term recording system containing inertial sensors (triaxial accelerometer, sample frequency $50 \mathrm{~Hz}$ ) with software developed to identify postural transitions and movements such as walking, standing, sitting, or lying [24, 35]. A walking period was defined as an interval with at least three successive steps [24]. Activities with less than three steps were considered as standing. Steps were estimated by the detection of an acceleration peak beyond a predefined threshold after using an appropriate filter [36]. The analysis algorithms for step detection and activity classification (walking, standing, sitting, and lying) are described in detail elsewhere $[24,35,36]$. The PAMSys is sensitive (87-99\%) and specific (87-99.7\%) for the detection of PA patterns in older adults and patient populations $[24,35]$.

PA parameters calculated from raw data included daily duration of walking, standing, sitting, and lying (as percentage). Further, specific PA parameters including duration of single walking, standing, sitting, lying episodes, the variability of these durations (expressed as standard deviation), number of steps, and duration of sit-to-stand as well as stand-to-sit transfers were calculated (table 1).

A standardized interviewer-administered questionnaire using the 5-point Likert scale was used to address acceptability of the PAMSys including comfort in wearing the shirt, awareness of the monitor, interference of the monitor with daily activities and sleep, adverse events, and adherence in wearing the monitor.

\section{Statistical Analysis}

An a priori power analysis was conducted for our first study aim (i.e., separating frailty status groups based on gait speed). A study of 100 community-dwelling elders aged $\geq 75$ years observed a mean gait speed $( \pm S D)$ of $1.24 \pm 0.13 \mathrm{~m} / \mathrm{s}$ in 25 nonfrail, $0.95 \pm$ $0.21 \mathrm{~m} / \mathrm{s}$ in 55 prefrail, and $0.80 \pm 0.19 \mathrm{~m} / \mathrm{s}$ in 20 frail subjects [9]. Assuming the same variability from this study and an alpha of $0.017(0.05 / 3$ to adjust for three pairwise comparisons), we would have $80 \%$ power to detect a difference of $0.2 \mathrm{~m} / \mathrm{s}$ with $\mathrm{n}=16$ per group for nonfrail versus prefrail, $n=21$ per group for prefrail versus frail, and $\mathrm{n}=14$ per group for nonfrail versus frail.

We used $\chi^{2}$ tests to evaluate differences in categorical demographic/clinical characteristics across frailty status groups (nonfrail, prefrail, and frail). Metric measures including both demographic/clinical and sensor data were compared (SPSS Statistics
Desktop, V22.0.0) using ANCOVA with the Games-Howell post hoc pairwise test (which controls for a family-wise type 1 error rate and is robust under unequal group sizes and unequal variances) to test for significant differences in each parameter between three frailty status groups. The discriminative power of each sensor-derived variable was calculated using Cohen's d effect sizes (e.g., d of 0.2 was considered as small, 0.5 as medium, and 0.8 as large).

We used logistic regression (Stata version 12) to evaluate sensor-based variables with a Cohen's d effect size of $\geq 0.5$ as potential screening measures for prefrailty. Sequential models estimated the prevalence odds ratios (ORs) for prefrail relative to nonfrail, both unadjusted and adjusted for age. The area under the curve (AUC) was calculated for estimating the predictive validity of each parameter. Variables with the highest AUC in each class (gait, balance, and PA) representing the most sensitive prefrailty screening parameters are presented in table 2 .

We used multinomial logistic regression (Stata version 12) [37], with reference group prefrail, to evaluate sensor-based variables that discriminate three frailty levels. The variables assessed had Cohen's d effect sizes $\geq 0.5$ for both nonfrail versus prefrail and prefrail versus frail. Sequential models estimated the prevalence ORs for prefrail relative to nonfrail (inverse OR presented) and frail relative to prefrail, both unadjusted and adjusted for age. The AUC for multinomial models was estimated using the 'MLOGITROC' command in Stata 12, which generates multiclass ROC curves for classification accuracy using bootstrapping methods and smoothed probability distributions derived from kernel density estimation [38].

\section{Results}

\section{Demographic and Clinical Characteristics}

A total of 125 individuals were included in the study in which $44(35.2 \%)$ were identified as nonfrail, 60 (48.0\%) as prefrail, and 21 (16.8\%) as frail according to the criteria established by Fried et al. [1]. Demographics and clinical characteristics are displayed in table 3. Compared to nonfrail individuals, prefrail and frail individuals were significantly older, took more medications, had higher BMI, more perceived tiredness, and greater fear of falling. Compared to prefrail individuals, frail individuals had significantly higher levels of depressive symptoms, greater fear of falling, more perceived tiredness, and lower ADL scores. There was an increase in use of assistive devices with increasing frailty level.

\section{Sensor-Based Gait Assessment}

For single-task walking speed, stride length and double support significantly discriminated between the three frailty status groups (table 4). The discriminatory power of these gait variables was higher for nonfrail versus prefrail individuals $(\mathrm{d}=0.93-1.18)$ compared to prefrail ver- 
Table 1. PA characteristics from a 24-hour motion sensor measurement stratified by frailty status

\begin{tabular}{|c|c|c|c|c|c|c|c|c|c|}
\hline \multirow[t]{2}{*}{ Variables } & \multirow[t]{2}{*}{ Nonfrail (N) } & \multirow[t]{2}{*}{ Prefrail (P) } & \multirow[t]{2}{*}{ Frail (F) } & \multicolumn{3}{|l|}{$\mathrm{p}$ value ${ }^{\mathrm{a}}$} & \multicolumn{3}{|c|}{ Effect size ${ }^{b}$} \\
\hline & & & & $\mathrm{N}$ vs. $\mathrm{P}$ & P vs. F & $\mathrm{N}$ vs. $\mathrm{F}$ & N vs. $\mathrm{P}$ & P vs. F & $\mathrm{N}$ vs. $\mathrm{F}$ \\
\hline \multicolumn{10}{|l|}{ Walking } \\
\hline Walking during $24 \mathrm{~h}, \%$ & $8.7 \pm 3.9$ & $6.1 \pm 3.0$ & $4.7 \pm 3.4$ & 0.001 & 0.283 & 0.001 & 0.75 & 0.44 & 1.09 \\
\hline Mean walking bout duration, s & $15.3 \pm 3.12$ & $14.3 \pm 2.7$ & $13.0 \pm 3.1$ & 0.201 & 0.257 & 0.030 & 0.35 & 0.45 & 0.75 \\
\hline Walking bout duration variability, s & $26.0 \pm 19.6$ & $17.2 \pm 13.3$ & $9.5 \pm 6.8$ & 0.026 & 0.015 & $<0.001$ & 0.52 & 0.73 & 1.12 \\
\hline Longest walking bout duration, s & $355 \pm 351$ & $212 \pm 253$ & $100 \pm 79$ & 0.063 & 0.012 & $<0.001$ & 0.47 & 0.59 & 1.01 \\
\hline Steps during $24 \mathrm{~h}, \mathrm{n}$ & $6,030 \pm 3,075$ & $3,869 \pm 1,996$ & $3,080 \pm 2,034$ & $<0.001$ & 0.411 & 0.001 & 0.83 & 0.36 & 1.08 \\
\hline Maximum continuous steps, $\mathrm{n}$ & $352 \pm 375$ & $183 \pm 220$ & $83 \pm 67$ & 0.029 & 0.008 & $<0.001$ & 0.55 & 0.62 & 1.00 \\
\hline \multicolumn{10}{|l|}{ Standing } \\
\hline Standing during $24 \mathrm{~h}, \%$ & $16.8 \pm 5.8$ & $14.3 \pm 4.6$ & $13.3 \pm 6.4$ & 0.066 & 0.764 & 0.120 & 0.48 & 0.18 & 0.57 \\
\hline Mean standing bout duration, s & $29.8 \pm 9.8$ & $31.8 \pm 7.0$ & $39.0 \pm 15.1$ & 0.466 & 0.148 & 0.061 & 0.24 & 0.61 & 0.73 \\
\hline Longest standing bout duration, s & $348 \pm 392$ & $514 \pm 813$ & $497 \pm 420$ & 0.540 & 0.992 & 0.600 & 0.26 & 0.03 & 0.37 \\
\hline Standing bout duration variability, s & $38.4 \pm 21.7$ & $47.5 \pm 45.8$ & $46.2 \pm 32.0$ & 0.388 & 0.802 & 0.132 & 0.25 & 0.03 & 0.28 \\
\hline \multicolumn{10}{|l|}{ Sitting } \\
\hline Sitting during $24 \mathrm{~h}, \%$ & $43.8 \pm 15.5$ & $53.3 \pm 13.2$ & $56.3 \pm 14.7$ & 0.005 & 0.745 & 0.016 & 0.66 & 0.21 & 0.83 \\
\hline Mean sitting bout duration, $\mathrm{s}$ & $373 \pm 189$ & $453 \pm 207$ & $572 \pm 273$ & 0.112 & 0.225 & 0.025 & 0.40 & 0.49 & 0.85 \\
\hline Longest sitting bout duration, s & $5,453 \pm 3,481$ & $6,181 \pm 2,866$ & $7,677 \pm 4,081$ & 0.503 & 0.333 & 0.125 & 0.23 & 0.42 & 0.59 \\
\hline Sitting bout duration variability, s & $774 \pm 451$ & $909 \pm 394$ & $1,048 \pm 686$ & 0.256 & 0.118 & 0.022 & 0.30 & 0.25 & 0.47 \\
\hline \multicolumn{10}{|l|}{ Lying } \\
\hline Lying during 24 h, \% & $30.6 \pm 15.6$ & $26.1 \pm 13.5$ & $25.8 \pm 15.6$ & 0.191 & 0.997 & 0.433 & 0.31 & 0.02 & 0.31 \\
\hline Mean lying bout duration, s & $1,837 \pm 1,321$ & $2,661 \pm 2,365$ & $1,559 \pm 1,384$ & 0.065 & 0.115 & 0.990 & 0.43 & 0.57 & 0.21 \\
\hline Longest lying bout duration, s & $11,366 \pm 11,491$ & $9,266 \pm 6,440$ & $7,592 \pm 5,865$ & 0.437 & 0.926 & 0.362 & 0.23 & 0.27 & 0.41 \\
\hline Lying bout duration variability, s & $3,098 \pm 2,491$ & $3,136 \pm 2,180$ & $2,355 \pm 1,806$ & 0.992 & 0.630 & 0.631 & 0.02 & 0.39 & 0.34 \\
\hline \multicolumn{10}{|l|}{ Transfers } \\
\hline Sit-stand duration, 90th percentile, $s$ & $3.87 \pm 0.68$ & $4.09 \pm 0.72$ & $4.46 \pm 0.78$ & 0.258 & 0.188 & 0.023 & 0.31 & 0.49 & 0.81 \\
\hline Stand-sit duration, 90th percentile, s & $3.87 \pm 0.68$ & $4.20 \pm 0.92$ & $4.73 \pm 0.93$ & 0.096 & 0.104 & 0.004 & 0.41 & 0.57 & 1.06 \\
\hline
\end{tabular}

The results of each derived PA parameter (mean \pm SD) for different frailty status groups are presented.

a Games-Howell contrasts due to unequal group sizes and in some cases unequal variances. ${ }^{b}$ Effect sizes have been calculated as Cohen's d.

Table 2. The most sensitive prefrailty screening variables from sensor-based gait, balance, and PA assessment

\begin{tabular}{|c|c|c|c|c|c|c|}
\hline \multirow[t]{2}{*}{ Variables } & \multicolumn{3}{|c|}{ Unadjusted model } & \multicolumn{3}{|c|}{ Age-adjusted model } \\
\hline & OR & $95 \% \mathrm{CI}$ & AUC & OR & $95 \% \mathrm{CI}$ & AUC \\
\hline Gait: speed, single-task, per $10 \mathrm{~cm} / \mathrm{s}$ & 0.53 & $0.40-0.71$ & 0.797 & 0.56 & $0.41-0.76$ & 0.802 \\
\hline Balance: hip sway, deg ${ }^{2}$ & 1.12 & $1.05-1.20$ & 0.647 & 1.11 & $1.03-1.19$ & 0.734 \\
\hline PA: steps during $24 \mathrm{~h}$, per 100 steps & 0.97 & $0.95-0.98$ & 0.712 & 0.97 & $0.96-0.99$ & 0.763 \\
\hline
\end{tabular}

sus frail individuals $(\mathrm{d}=0.70-0.85)$. Gait speed best discriminated nonfrail versus prefrail individuals $(\mathrm{d}=1.18)$, whereas stride length best discriminated prefrail versus frail $(\mathrm{d}=0.85)$. Discriminatory power of gait variables was lower for dual-task gait assessment $(\mathrm{d}=0.20-1.46)$ when compared to single-task gait assessment $(\mathrm{d}=0.24-$ 1.64). In this study, we found that subjects who used assistive devices had a slower gait speed than those without devices, and these differences increased with increasing frailty level.

\section{Sensor-Based Balance Assessment}

The results of the sensor-based balance assessment are presented in table 4 . Balance parameters discriminated between nonfrail and prefrail individuals, with hip sway as the best discriminator $(d=0.62)$, but not between prefrail and frail individuals $(\mathrm{p}=0.653-0.999)$.

\section{Sensor-Based PA Assessment}

Among the five PA categories measured (i.e., walking, standing, sitting, lying, and transfers), parameters related 
Table 3. Demographic and clinical characteristics

\begin{tabular}{|c|c|c|c|c|c|c|}
\hline \multirow[t]{2}{*}{ Characteristics } & \multirow{2}{*}{$\begin{array}{l}\text { Nonfrail }(\mathrm{N}) \\
(\mathrm{n}=44)\end{array}$} & \multirow{2}{*}{$\begin{array}{l}\text { Prefrail (P) } \\
(n=60)\end{array}$} & \multirow{2}{*}{$\begin{array}{l}\text { Frail (F) } \\
(n=21)\end{array}$} & \multicolumn{3}{|l|}{$\mathrm{p}$ value $^{\mathrm{a}}$} \\
\hline & & & & $\mathrm{N}$ vs. $\mathrm{P}$ & $\mathrm{P}$ vs. F & $\mathrm{N}$ vs. $\mathrm{F}$ \\
\hline Age, years & $74.6 \pm 6.5$ & $80.2 \pm 8.6$ & $83.4 \pm 8.6$ & 0.001 & 0.330 & 0.001 \\
\hline \multicolumn{7}{|l|}{ Sex } \\
\hline Female & $37(84.1)$ & $45(75.0)$ & $18(85.7)$ & 0.262 & 0.376 & 0.999 \\
\hline Male & $7(15.9)$ & $15(25.0)$ & $3(14.3)$ & & & \\
\hline \multicolumn{7}{|l|}{ Race } \\
\hline White & $37(84.1)$ & $50(83.3)$ & $16(76.2)$ & 0.957 & 0.352 & 0.082 \\
\hline African American & $0(0.0)$ & $1(1.7)$ & $2(9.5)$ & & & \\
\hline American Indian & $0(0.0)$ & $1(1.7)$ & $1(4.8)$ & & & \\
\hline Asian & $0(0.0)$ & $1(1.7)$ & $0(0.0)$ & & & \\
\hline Other & $7(15.9)$ & $7(11.7)$ & $2(9.5)$ & & & \\
\hline History of falls & $13(33.3)$ & $26(47.3)$ & $11(57.9)$ & 0.177 & 0.425 & 0.075 \\
\hline Use of assistance devices ${ }^{b}$ & $4(10.0)$ & $26(47.3)$ & $14(73.7)$ & $<0.001$ & 0.046 & $<0.001$ \\
\hline Number of prescriptions & $2.5 \pm 1.8$ & $4.1 \pm 3.8$ & $6.0 \pm 3.4$ & 0.021 & 0.138 & 0.001 \\
\hline \multicolumn{7}{|l|}{ Number of social activities } \\
\hline 0 & $1(2.9)$ & $3(7.3)$ & $2(13.3)$ & 0.079 & 0.025 & $<0.001$ \\
\hline $1-3$ & $14(40.0)$ & $25(61.0)$ & $13(86.7)$ & & & \\
\hline$>3$ & $20(57.1)$ & $13(31.7)$ & $0(0.0)$ & & & \\
\hline \multicolumn{7}{|l|}{ BMI } \\
\hline$<25$ & $24(54.6)$ & $19(31.7)$ & $4(19.1)$ & 0.013 & 0.251 & 0.012 \\
\hline $25-29.9$ & $12(27.3)$ & $13(21.7)$ & $9(42.9)$ & & & \\
\hline $30-34.9$ & $7(15.9)$ & $18(30.0)$ & $4(19.1)$ & & & \\
\hline$\geq 35$ & $1(2.3)$ & $10(16.7)$ & $4(19.1)$ & & & \\
\hline MMSE & $29.2 \pm 1.1$ & $28.6 \pm 1.6$ & $28.7 \pm 1.7$ & 0.121 & 0.980 & 0.531 \\
\hline Mobility-Tiredness Scale & $5.6 \pm 0.8$ & $4.7 \pm 1.4$ & $2.7 \pm 1.8$ & $<0.001$ & $<0.001$ & $<0.001$ \\
\hline CES-D & $6.6 \pm 5.7$ & $6.9 \pm 6.8$ & $14.0 \pm 7.0$ & 0.958 & 0.001 & 0.001 \\
\hline Falls Efficacy Scale-International & $20.8 \pm 4.2$ & $28.0 \pm 9.5$ & $39.5 \pm 12.3$ & $<0.001$ & 0.002 & $<0.001$ \\
\hline Barthel ADL Scale & $95.5(14.0)$ & $96.2(5.5)$ & $88.1(9.0)$ & 0.955 & 0.002 & 0.033 \\
\hline
\end{tabular}

Values are presented as means $\pm \mathrm{SD}$ or $\mathrm{n}(\%) .{ }^{\text {a }}$ Games-Howell contrasts for continuous measures due to unequal group sizes and in some cases unequal variances. ${ }^{\mathrm{b}}$ Canes and walkers.

to walking best discriminated between nonfrail and prefrail individuals, with highest effect sizes found for number of steps $(d=0.83)$ and percentage of walking $(d=$ 0.75) (table 1). Furthermore, prefrail adults took significantly fewer continuous steps $(\mathrm{d}=0.55)$. Interestingly, the variability of walking bout duration was significantly lower in prefrail compared to nonfrail individuals $(\mathrm{d}=$ 0.52 ). Besides lower amounts of walking activity, prefrail adults had an increased percentage of sitting $(\mathrm{d}=0.66)$ when compared to nonfrail adults.

Importantly, percentage of walking $(\mathrm{p}=0.283)$ was a poor discriminator compared to other walking parameters, including the longest walking bout duration $(\mathrm{d}=0.59)$, maximum continuous steps $(\mathrm{d}=0.62)$, and variability of walking bouts $(\mathrm{d}=0.73)$, which significantly discriminated between prefrail and frail individuals.

Wearable Sensor-Based Assessment of Frailty
The acceptability of wearing the T-shirt-embedded motion sensor over a 24 -hour period was high. For each question, the majority of subjects (66-98\%) strongly or somewhat agreed to positive attributes, with no significant differences by frailty status groups (nonfrail 66-98\%; prefrail 68-98\%; frail 67-95\%; $\mathrm{p}=0.13-1.00$ ) (online suppl. table, www.karger.com/doi/10.1159/000369095).

\section{The Most Sensitive Variables for Prefrailty Screening}

The most sensitive prefrailty screening variables of each domain (gait, balance, and PA) are presented in table 2 . Single-task walking speed had the highest validity for the identification of prefrailty ( $A U C=0.802)$. Among PA parameters, the number of steps $(\mathrm{AUC}=0.763)$ emerged as the most sensitive parameter for prefrailty screening. The predictive validity of balance parameters (hip sway, $A U C=0.734)$ was inferior when compared to gait and PA. 
Table 4. Results of the gait and balance assessment stratified by frailty status

\begin{tabular}{|c|c|c|c|c|c|c|c|c|c|}
\hline \multirow[t]{2}{*}{ Gait parameters } & \multirow[t]{2}{*}{ Nonfrail (N) } & \multirow[t]{2}{*}{ Prefrail (P) } & \multirow[t]{2}{*}{ Frail (F) } & \multicolumn{3}{|l|}{$\mathrm{p}$ value $^{\mathrm{a}}$} & \multicolumn{3}{|c|}{ Effect size ${ }^{b}$} \\
\hline & & & & $\mathrm{N}$ vs. $\mathrm{P}$ & P vs. F & $\mathrm{N}$ vs. F & $\mathrm{N}$ vs. $\mathrm{P}$ & P vs. F & $\mathrm{N}$ vs. $\mathrm{F}$ \\
\hline \multicolumn{10}{|l|}{ Single-task walking } \\
\hline Speed, $\mathrm{m} / \mathrm{s}$ & $1.17 \pm 0.15$ & $0.94 \pm 0.23$ & $0.71 \pm 0.36$ & $<0.001$ & 0.033 & $<0.001$ & 1.18 & 0.76 & 1.55 \\
\hline Stride time, s & $1.08 \pm 0.10$ & $1.20 \pm 0.19$ & $1.33 \pm 0.22$ & 0.007 & 0.064 & $<0.001$ & 0.79 & 0.63 & 1.43 \\
\hline Stride length, $\mathrm{m}$ & $1.25 \pm 0.11$ & $1.09 \pm .0 .18$ & $0.88 \pm 0.30$ & 0.005 & 0.015 & $<0.001$ & 1.07 & 0.85 & 1.64 \\
\hline Double support, $\%$ & $22.18 \pm 4.18$ & $27.19 \pm 6.39$ & $32.29 \pm 8.11$ & $<0.001$ & 0.043 & $<0.001$ & 0.93 & 0.70 & 1.56 \\
\hline CV stride velocity, \% & $4.81 \pm 3.42$ & $5.76 \pm 4.31$ & $7.15 \pm 4.24$ & 0.507 & 0.449 & 0.181 & 0.24 & 0.33 & 0.61 \\
\hline \multicolumn{10}{|l|}{ Dual-task waking } \\
\hline Speed, $\mathrm{m} / \mathrm{s}$ & $1.06 \pm 0.19$ & $0.86 \pm 0.25$ & $0.65 \pm 0.35$ & 0.005 & 0.052 & $<0.001$ & 0.90 & 0.69 & 1.46 \\
\hline Stride time, $s$ & $1.19 \pm 0.17$ & $1.34 \pm 0.25$ & $1.44 \pm 0.28$ & 0.002 & 0.297 & 0.002 & 0.70 & 0.38 & 1.07 \\
\hline Stride length, $\mathrm{m}$ & $1.23 \pm 0.14$ & $1.10 \pm 0.21$ & $0.87 \pm 0.32$ & $<0.001$ & 0.018 & $<0.001$ & 0.73 & 0.85 & 1.46 \\
\hline Double support, $\%$ & $24.01 \pm 4.95$ & $29.5 \pm 7.14$ & $35.86 \pm 11.02$ & $<0.001$ & 0.059 & $<0.001$ & 0.89 & 0.68 & 1.39 \\
\hline CV stride velocity, \% & $6.50 \pm 3.76$ & $7.27 \pm 3.91$ & $9.59 \pm 5.64$ & 0.601 & 0.223 & 0.085 & 0.20 & 0.48 & 0.64 \\
\hline \multicolumn{10}{|l|}{ Balance parameter } \\
\hline Ankle sway, deg $^{2}$ & $6.15 \pm 4.60$ & $9.46 \pm 9.46$ & $13.28 \pm 17.98$ & 0.057 & 0653 & 0.229 & 0.45 & 0.27 & 0.54 \\
\hline Hip sway, $\operatorname{deg}^{2}$ & $5.91 \pm 3.44$ & $12.12 \pm 13.76$ & $12.21 \pm 16.59$ & 0.004 & 0.999 & 0.254 & 0.62 & 0.01 & 0.53 \\
\hline Mean COM sway, $\mathrm{cm}^{2}$ & $1.20 \pm 0.83$ & $2.07 \pm 2.20$ & $2.22 \pm 2.24$ & 0.021 & 0.964 & 0.159 & 0.51 & 0.06 & 0.58 \\
\hline COM ML sway, cm & $0.75 \pm 0.36$ & $0.92 \pm 0.62$ & $0.93 \pm 0.50$ & 0.203 & 0.999 & 0.371 & 0.33 & 0.03 & 0.42 \\
\hline COM AP sway, cm & $1.51 \pm 0.70$ & $1.90 \pm 1.03$ & $2.07 \pm 1.09$ & 0.063 & 0.812 & 0.114 & 0.44 & 0.17 & 0.62 \\
\hline Ankle sway, deg ${ }^{2}$ & $6.15 \pm 4.60$ & $9.46 \pm 9.46$ & $13.28 \pm 17.98$ & 0.057 & 0.653 & 0.229 & 0.45 & 0.27 & 0.54 \\
\hline
\end{tabular}

Spatio-temporal parameters (mean \pm SD) derived from gait assessment (separately for single- and dual-task walking) and sway parameters derived from balance assessment for different frailty status groups are presented.

${ }^{a}$ Games-Howell contrasts due to unequal group sizes and in some cases unequal variances.

b Effect sizes have been calculated as Cohen's d.

Table 5. The most sensitive parameters for discriminating three frailty levels (nonfrail, prefrail, and frail)

\begin{tabular}{|c|c|c|c|c|c|c|c|c|c|c|}
\hline \multirow[t]{3}{*}{ Variables } & \multicolumn{5}{|c|}{ Unadjusted model } & \multicolumn{5}{|c|}{ Age-adjusted model ${ }^{\mathrm{a}}$} \\
\hline & \multicolumn{2}{|c|}{ Prefrail vs. nonfrail } & \multicolumn{3}{|c|}{ Frail vs. prefrail } & \multicolumn{2}{|c|}{ Prefrail vs. nonfrail } & \multicolumn{3}{|c|}{ Frail vs. prefrail } \\
\hline & OR & $95 \% \mathrm{CI}$ & OR & $95 \% \mathrm{CI}$ & AUC & OR & $95 \% \mathrm{CI}$ & OR & $95 \% \mathrm{CI}$ & AUC \\
\hline Gait: stride length, single-task, per $10 \mathrm{~cm}$ & 0.52 & $0.36-0.74$ & 0.65 & $0.50-0.86$ & 0.853 & 0.57 & $0.39-0.83$ & 0.63 & $0.44-0.92$ & 0.857 \\
\hline Gait: double support, single-task, \% & 1.22 & $1.11-1.34$ & 1.11 & $1.02-1.20$ & 0.776 & 1.21 & $1.10-1.34$ & 1.10 & $1.003-1.20$ & 0.841 \\
\hline Gait: speed, single task, per $10 \mathrm{~cm} / \mathrm{s}$ & 0.59 & $0.43-0.80$ & 0.71 & $0.54-0.94$ & 0.796 & 0.63 & $0.46-0.87$ & 0.71 & $0.50-1.007$ & 0.830 \\
\hline PA: walking bout duration variability, $s$ & 0.96 & $0.93-0.99$ & 0.91 & $0.83-0.99$ & 0.814 & 0.97 & $0.93-1.002$ & 0.90 & $0.83-0.99$ & 0.818 \\
\hline
\end{tabular}

Multinomial logistic regression with the prefrail group as reference. Inverse ORs presented for prefrail vs. nonfrail.

${ }^{a}$ Adjustment for age did not appreciably alter significance, except for gait speed (frail vs. prefrail, $\mathrm{p}=0.055$ ) walking bout duration variability (prefrail vs. nonfrail, $\mathrm{p}=0.065)$.

\section{The Most Sensitive Variables for Discriminating between Three Frailty Levels}

The results of the multinomial logistic regression analysis for evaluating the ability of parameters to discriminate between the three frailty levels are presented in table 5. Among gait parameters, stride length (AUC = $0.857)$ and double support $(\mathrm{AUC}=0.841)$ had the highest validity to separate both nonfrail from prefrail individuals and prefrail from frail individuals in the age-adjusted model. A smaller AUC was obtained for gait speed (0.830), and the ability of this parameter to separate prefrail versus frail individuals became nonsignificant after adjusting for age $(p=0.055)$. Interestingly, walking bout duration variability emerged as the most sensitive PA parameter to 
separate the three frailty groups (AUC 0.818 ), although the ability to separate nonfrail versus prefrail individuals became nonsignificant $(\mathrm{p}=0.065)$ after adjusting for age. No other gait, balance, or PA parameter was able to discriminate between the three groups simultaneously.

\section{Discussion}

To our knowledge, this is the first study that compared multiple instrumented assessments for quantifying physical function and PA across levels of frailty in a home and community environment. Gait parameters were found to be the most sensitive for the identification of a subject's level of frailty. Additionally, specific PA parameters emerged as sensitive indicators of the frailty level, suggesting that continuous monitoring of everyday activities may be a valid and autonomous method for the identification of frailty.

\section{Sensor-Based Gait Assessment}

Gait speed was the most sensitive parameter for the identification of prefrailty. The results are in accordance with findings from a systematic review, which reports gait speed as the best discriminator between nonfrail and prefrail individuals among different gait parameters [11]. It should be noted that the Fried frailty criteria include slow gait speed [1], which explains the high discriminative power of this variable. In the present study, the discriminative ability of gait speed can be used as a reference for comparison with other parameters analyzed.

Interestingly, results suggest that stride length and double support are more sensitive for classifying frailty level compared to gait speed. Reduced stride length is linked to a lack of lower extremity strength [39] and may be an indicator of frailty-associated sarcopenia [40]. Increased double support time is an attempt to minimize postural instability [41] and may indicate deficits in dynamic balance control associated with frailty. Our findings suggest that specific gait parameters can quantify frailty-related aspects including loss of muscle mass and balance and may add precision to gait-based frailty screening. Notably, a systematic review identified the same gait characteristics (i.e., stride length, double support) as the most sensitive discriminators between prefrail and frail individuals [11] among different gait parameters.

Previous studies have reported that adding a cognitively demanding task to gait assessment ('dual tasking') is sensitive in identifying individuals who may be at risk of developing frailty [19]. In the present study, we did not find any benefit for dual-task gait assessment, which is likely related to the fact that we excluded individuals with cognitive impairment. In addition, the walking distance used in this study $(4.57 \mathrm{~m})$ may not be sufficient to address gait alteration due to dual tasking $[42,43]$.

\section{Sensor-Based Balance Assessment}

The present findings suggest that a balance deficit is a specific marker of prefrailty. These results are in accordance with previous studies, which found significant differences in postural balance between the nonfrail and prefrail/frail groups, but not between the prefrail and frail ones $[44,45]$. Our results show that hip sway is a more sensitive marker of prefrailty compared to ankle sway. This may suggest that the prefrail individuals used a 'hip strategy' rather than an 'ankle strategy' to compensate for threatened balance (when standing with eyes closed). Previous studies in older adults suggest that the use of a 'hip strategy' is related to a loss of peripheral somatosensation and/or weakness in ankle joint muscles [46]; this strategy is associated with an increased fall risk [47].

\section{Sensor-Based PA Assessment}

Our results show that the PA level is reduced with increasing frailty, which was expected because low PA (based on self-report) is a criterion of the Fried frailty index. On the same note, previous studies have not objectively evaluated the specific impact of frailty on everyday PA characteristics. Results of our sensor-based PA assessment may suggest that different frailty stages can be discriminated by specific PA variables. Nonfrail and prefrail individuals were best discriminated by a decline in number of steps, as reported previously [21]. In contrast, differences between prefrail and frail were characterized by other, more specific, PA characteristics such as reductions in the longest walking bout duration. We speculate that the drastic reduction in longest walking bout duration $(-53 \%)$ in frail (compared to prefrail) individuals is related to impairments in physical function and/or exhaustion, both key indicators of frailty [1]. Our results suggest that the sensor-based PA assessment can quantify frailty-associated loss of everyday PA, which may help to better estimate the level of frailty and its impact on daily functioning. Interestingly, we observed a continuous reduction in the variability of walking episodes with increasing frailty level. Our findings indicate more static and less complex PA behavior in frail individuals, characterized predominately by short walking bouts. Our results may suggest that the 'loss of complexity paradigm' related to frailty [26] is reflected not only by physiologic systems but also by everyday PA behavior. 


\section{Limitations and Future Research}

Participants of this study were recruited using a convenience sample technique; thus, the sample may not represent the general population of community-dwelling older adults. The proposed parameters derived from our exploratory analysis must be validated in a larger nonselected sample to evaluate their true predictive potential. However, because we saw participants in both home and community settings, we were able to include nearlyhomebound subjects in our study, who are often excluded in clinic-based studies.

We included assistive device users (canes and walkers), although walking aids may minimize the detection of gait deficits [48]. Despite walking aid usage, gait variables had the highest discriminative power, suggesting that walking aids did not substantially impact on gaitbased frailty screening. The order of the single and dual tasks was not randomized or counter-balanced, which may have biased the results of dual-task walking due to effects of practice or fatigue.

The 24-hour PA assessment period did not cover dayto-day variability, although PA in older adults is less variable than in younger populations and day-to-day reliability of PA assessment was high in a sample of older adults (>60 years) [49]. The 24-hour monitoring in our study may therefore have been sufficient to document habitual PA because of low day-to-day variability. However, further research should address whether a longer period of monitoring increases the accuracy of frailty screening.

Multiple frailty concepts have been proposed and no consensus about an operative definition exists [50]. In this study, we have used the most widely adopted Fried frailty criteria, which are associated with gait, sarcopenia, and PA. Thus, our presented sensor approach of measuring frailty might be overoptimistic then when employing a broader frailty concept including cognitive, psycholog- ical, or social components, or a cumulative concept such as the Rockwood model [51]. Future studies should validate the objective parameters identified in this study using alternative frailty definitions.

The cross-sectional design of this study limits inferences of causality. The next step will be a longitudinal analysis using 6-month follow-up data from the same study participants allowing determination of the extent to which different parameters predict changes in frailty status. This may allow the development of interventions targeted to discover decrements.

\section{Conclusions}

We found that objective gait, balance, and PA parameters have the potential to provide clinically meaningful surveillance of older adults across frailty statuses. Particularly, our approach of monitoring frailty-specific PA parameters may be incorporated into $\mathrm{mHealth}$ technologies (i.e., smartphone) and may serve as a 'frailty meter', similar to a Holter monitor. These are steps towards an objective screening tool for the identification of clinical frailty syndrome in older adults.

\section{Acknowledgements}

We thank the Arizona Frailty Cohort coordination staff and the student workers for data collection and participant recruitment. This study was supported in part by award No. 2R42AG032748 from the National Institute on Aging.

\section{Disclosure Statement}

The authors have no financial or personal conflicts of interest to declare.

\section{References}

1 Fried LP, Tangen CM, Walston J, Newman AB, Hirsch C, Gottdiener J, Seeman T, Tracy R, Kop WJ, Burke G, McBurnie MA; Cardiovascular Health Study Collaborative Research Group: Frailty in older adults: evidence for a phenotype. J Gerontol A Biol Sci Med Sci 2001; 56:M146-M156.

2 Fassbender K, Fainsinger RL, Carson M, Finegan BA: Cost trajectories at the end of life: the Canadian experience. J Pain Symptom Manage 2009;38:75-80.

3 da Câmara SMA, Alvarado BE, Guralnik JM, Guerra RO, Maciel ÁCC: Using the Short Phys- ical Performance Battery to screen for frailty in young-old adults with distinct socioeconomic conditions. Geriatr Gerontol Int 2013;13:421428

4 Kiely DK, Cupples LA, Lipsitz LA: Validation and comparison of two frailty indexes: The MOBILIZE Boston Study. J Am Geriatr Soc 2009;57:1532-1539.

5 Melzer D, Lan T-Y, Tom BD, Deeg DJ, Guralnik JM: Variation in thresholds for reporting mobility disability between national population subgroups and studies. J Gerontol A Biol Sci Med Sci 2004;59:1295-1303. 
6 Tudor-Locke C, Myers A: Challenges and opportunities for measuring physical activity in sedentary adults. Sports Med 2001;31:91-100.

7 Davis DH, Rockwood MR, Mitnitski AB, Rockwood K: Impairments in mobility and balance in relation to frailty. Arch Gerontol Geriatr 2011;53:79-83.

8 Mohler MJ, Fain MJ, Wertheimer AM, Najafi B, Nikolich-Žugich J: The frailty syndrome: clinical measurements and basic underpinnings in humans and animals. Exp Gerontol 2014;54C:6-13.

$\checkmark 9$ Montero-Odasso M, Muir SW, Hall M, Doherty TJ, Kloseck M, Beauchet O, Speechley M: Gait variability is associated with frailty in community-dwelling older adults. J Gerontol A Biol Sci Med Sci 2011;66:568-576.

- 10 Saliba D, Elliott M, Rubenstein LZ, Solomon DH, Young RT, Kamberg CJ, Roth C, MacLean $\mathrm{CH}$, Shekelle PG, Sloss EM: The vulnerable elders survey: a tool for identifying vulnerable older people in the community. J Am Geriatr Soc 2001;49:1691-1699.

11 Schwenk M, Howe C, Saleh A, Mohler J, Grewal G, Armstrong D, Najafi B: Frailty and technology: a systematic review of gait analysis in those with frailty. Gerontology 2013;60:79-89.

${ }_{12}$ Verghese J, Xue X: Identifying frailty in high functioning older adults with normal mobility. Age Ageing 2010;39:382-385.

13 Kellen E, Bulens P, Deckx L, Schouten H, Van Dijk M, Verdonck I, Buntinx F: Identifying an accurate pre-screening tool in geriatric oncology. Crit Rev Oncol Hematol 2010;75:243-248.

14 Puthoff ML: Outcome measures in cardiopulmonary physical therapy: short physical performance battery. Cardiopulm Phys Ther J 2008 19:17.

15 Najafi B, Horn D, Marclay S, Crews RT, Wu S, Wrobel JS: Assessing postural control and postural control strategy in diabetes patients using innovative and wearable technology. J Diabetes Sci Technol 2010;4:780-791.

16 Rothman MD, Leo-Summers L, Gill TM: Prognostic significance of potential frailty criteria. J Am Geriatr Soc 2008;56:2211-2216.

-17 Beauchet O, Dubost V, Gonthier R, Kressig RW: Dual-task-related gait changes in transitionally frail older adults: the type of the walking-associated cognitive task matters. Gerontology 2005;51:48-52.

-18 Kressig RW, Gregor RJ, Oliver A, Waddell D, Smith W, O'Grady M, Curns AT, Kutner M, Wolf SL: Temporal and spatial features of gait in older adults transitioning to frailty. Gait Posture 2004;20:30-35.

$\checkmark 19$ Verghese J, Holtzer R, Lipton RB, Wang C: Mobility stress test approach to predicting frailty, disability, and mortality in high-functioning older adults. J Am Geriatr Soc 2012;60:19011905.

20 Peterson MJ, Giuliani C, Morey MC, Pieper CF, Evenson KR, Mercer V, Cohen HJ, Visser M, Brach JS, Kritchevsky SB: Physical activity as a preventative factor for frailty: the health, aging, and body composition study. J Gerontol A Biol Sci Med Sci 2009;64:61-68.

-21 Theou O, Jakobi JM, Vandervoort AA, Jones GR: A comparison of physical activity (PA) as- sessment tools across levels of frailty. Arch Gerontol Geriatr 2012;54:307-314.

22 Jorstad-Stein EC, Hauer K, Becker C, Bonnefoy M, Nakash RA, Skelton DA, Lamb SE: Suitability of physical activity questionnaires for older adults in fall-prevention trials: a systematic review. J Aging Phys Act 2005;13:461-481.

23 Hauer K, Lord SR, Lindemann U, Lamb SE, Aminian K, Schwenk M: Assessment of physical activity in older people with and without cognitive impairment. J Aging Phys Act 2011; 19:347-372.

24 Najafi B, Aminian K, Paraschiv-Ionescu A, Loew F, Bula CJ, Robert P: Ambulatory system for human motion analysis using a kinematic sensor: monitoring of daily physical activity in the elderly. IEEE Trans Biomed Eng 2003;50: 711-723.

25 Schwenk M, Hauer K, Zieschang T, Englert S, Mohler J, Najafi B: Sensor-derived physical activity parameters can predict future falls in people with dementia. Gerontology 2014;60:483492.

26 Lipsitz LA: Physiological complexity, aging, and the path to frailty. Sci Aging Knowledge Environ 2004:pe16.

27 Aminian K, Najafi B, Büla C, Leyvraz PF, Robert P: Spatio-temporal parameters of gait measured by an ambulatory system using miniature gyroscopes. J Biomech 2002;35:689-699.

28 Najafi B, Khan T, Wrobel J: Laboratory in a box: wearable sensors and its advantages for gait analysis. Conf Proc IEEE Eng Med Biol Soc 2011;2011:6507-6510.

29 Folstein MF, Folstein SE, McHugh PR: 'Minimental state'. A practical method for grading the cognitive state of patients for the clinician. J Psychiatr Res 1975;12:189-198.

30 Fieo RA, Mortensen EL, Rantanen T, Avlund $\mathrm{K}$ : Improving a measure of mobility-related fatigue (the mobility-tiredness scale) by establishing item intensity. J Am Geriatr Soc 2013; 61:429-433.

31 Eaton W, Smith C, Ybarra M, Muntaner C, Tien A: Center for Epidemiologic Studies Depression Scale: review and revision; in Maruish M (ed): The Use of Psychological Testing for Treatment Planning and Outcomes Assessment. New Jersey, Lawrence Erlbaum Associates, 2004, vol 3, pp 363-377.

-32 Yardley L, Beyer N, Hauer K, Kempen G, PiotZiegler C, Todd C: Development and initial validation of the Falls Efficacy Scale-International (FES-I). Age Ageing 2005;34:614-619.

33 Mahoney FI, Barthel DW: Functional evaluation: the Barthel index. Md State Med J 1965;14: 61-65.

34 Taylor HL, Jacobs DR Jr, Schucker B, Knudsen J, Leon AS, Debacker G: A questionnaire for the assessment of leisure time physical activities. J Chron Dis 1978;31:741-755.

35 Najafi B, Aminian K, Loew F, Blanc Y, Robert PA: Measurement of stand-sit and sit-stand transitions using a miniature gyroscope and its application in fall risk evaluation in the elderly. IEEE Trans Biomed Eng 2002;49:843-851.

36 Najafi B, Armstrong DG, Mohler J: Novel wearable technology for assessing spontaneous daily physical activity and risk of falling in older adults with diabetes. J Diabetes Sci Technol 2013;7:1147-1160.

37 Hosmer DW Jr, Lemeshow S: Applied Logistic Regression. New York, John Wiley \& Sons, 2004.

38 Peterson LE: Mlogitroc: Stata module to calculate multiclass ROC curves and AUC from multinomial logistic regression. Statistical Software Components S457181, Boston College Department of Economics, Boston, 2010.

39 Judge JO, Davis RB 3rd, Ônpuu S: Step length reductions in advanced age: the role of ankle and hip kinetics. J Gerontol A Biol Sci Med Sci 1996;51:M303-M312.

40 Marzetti E, Leeuwenburgh C: Skeletal muscle apoptosis, sarcopenia and frailty at old age. Exp Gerontol 2006;41:1234-1238.

41 Woollacott MH, Tang P-F: Balance control during walking in the older adult: research and its implications. Phys Ther 1997;77:646660

42 Lindemann U, Najafi B, Zijlstra W, Hauer K, Muche R, Becker C, Aminian K: Distance to achieve steady state walking speed in frail elderly persons. Gait Posture 2008;27:91-96.

43 Najafi B, Helbostad JL, Moe-Nilssen R, Zijlstra W, Aminian K: Does walking strategy in older people change as a function of walking distance? Gait Posture 2009;29:261-266.

-44 Dayhoff NE, Suhrheinrich J, Wigglesworth J, Topp R, Moore S: Balance and muscle strength as predictors of frailty among older adults. J Gerontol Nurs 1998;24:18-27.

-45 Martínez-Ramírez A, Lecumberri P, Gómez M, Rodriguez-Mañas L, García F, Izquierdo M: Frailty assessment based on wavelet analysis during quiet standing balance test. J Biomech 2011;44:2213-2220.

46 Woollacott $\mathrm{MH}$ : Changes in postural control and the integration of postural responses into voluntary movements with aging; in Brand $\mathrm{T}$, Paulus W, Bles W, Deiterich M, Krafezyk S, Strabue A (eds): Disorders of Posture and Gait. Stuttgart, Georg Thieme Verlag, 1990, pp 221228 .

47 Horak FB, Shupert CL, Mirka A: Components of postural dyscontrol in the elderly: a review. Neurobiol Aging 1989;10:727-738.

48 Schwenk M, Schmidt M, Pfisterer M, Oster P, Hauer K: Rollator use adversely impacts on assessment of gait and mobility during geriatric rehabilitation. J Rehabil Med 2011;43:424429.

49 Rowe DA, Kemble CD, Robinson TS, Mahar MT: Daily walking in older adults: day-to-day variability and criterion-referenced validity of total daily step counts. J Phys Act Health 2007; 4:434-446.

50 Rodríguez-Mañas L, Féart C, Mann G, Viña J, Chatterii S, Chodzko-Zajko W, Harmand MGC, Bergman H, Carcaillon L, Nicholson C: Searching for an operational definition of frailty: a Delphi method based consensus statement: the frailty operative definition-consensus conference project. J Gerontol A Biol Sci Med Sci 2013;68:62-67.

51 Rockwood K, Mitnitski A: Frailty in relation to the accumulation of deficits. J Gerontol A Biol Sci Med Sci 2007;62:722-727.
Wearable Sensor-Based Assessment of Frailty 